\title{
The lower the eosinophils, the stronger the inflammatory response? The relationship of different levels of eosinophils with the degree of inflammation in acute exacerbation chronic obstructive pulmonary disease (AECOPD)
}

\author{
Mei-Yu Lv, Li-Xia Qiang, Zhi-Heng Li, Shou-De Jin \\ Department of Respiratory Medicine, The Fourth Affiliated Hospital of Harbin Medical University, Harbin, China \\ Contributions: (I) Conception and design: MY Lv; (II) Administrative support: The Fourth Affiliated Hospital of Harbin Medical University; (III) \\ Provision of study materials or patients: None; (IV) Collection and assembly of data: MY Lv; (V) Data analysis and interpretation: MY Lv, LX Qiang, \\ ZH Li; (VI) Manuscript writing: All authors; (VII) Final approval of manuscript: All authors. \\ Correspondence to: Shou-De Jin. Department of Respiratory Medicine, The Fourth Affiliated Hospital of Harbin Medical University, No. 37 Yiyuan \\ Street, Nangang District, Harbin 150001, China. Email: jinshoude@163.com.
}

\begin{abstract}
Background: Blood eosinophil levels are a known marker for the effects of therapy in patients with chronic obstructive pulmonary disease (COPD). This study aimed to clarify the cutoff values for blood eosinophils (EOS) to predict exacerbation risk and prognosis of acute exacerbation COPD (AECOPD) and investigate their correlation using inflammatory indicators and clinical characteristics.

Methods: In this observational study of 174 patients with AECOPD, we assessed the relationship between EOS and COPD. According to the percentage of blood EOS, patients were grouped into two groups (Group 1: $\mathrm{EOS}<2 \%, \mathrm{n}=98$; Group 2: $\mathrm{EOS} \geq 2 \%, \mathrm{n}=76$ ), and Group 2 was further divided into Group A ( $\% \leq \mathrm{EOS}$ $<4 \%$ ) and Group B (EOS $\geq 4 \%$ ) based on a cutoff value of $4 \%$. Patients received standardized treatment after collection of peripheral blood specimen. Associations of EOS with laboratory indicators before any treatment in hospital and with clinical data were compared.

Results: Patients in Group 1 showed significantly severe inflammation, worse pulmonary function, longer length of stay (LOS) $(\mathrm{P}<0.001)$, higher $\mathrm{mMRC}$ score $(\mathrm{P}<0.05)$, higher CAT score $(\mathrm{P}<0.05)$, higher rates of mortality $(\mathrm{P}<0.05)$, and greater noninvasive mechanical ventilation usage $(\mathrm{P}<0.05)$ compared with Group 2 . Intriguingly, the $\mathrm{CRP}$, total $\mathrm{mMRC}$ and CAT scores of patients in Group A were significantly lower than those in Group $\mathrm{B}(\mathrm{P}<0.001 ; \mathrm{P}<0.01$; $\mathrm{P}<0.05$, respectively). Pearson correlation analysis showed that a low percentage blood eosinophil level was negatively associated with higher WBC count $(\mathrm{r}=-0.155, \mathrm{P}<0.05), \mathrm{NLR}(\mathrm{r}=-0.227, \mathrm{P}<0.01)$ and $\mathrm{CRP}(\mathrm{r}=-0.308, \mathrm{P}<0.01)$.

Conclusions: Different cutoff values for percentage blood EOS might be useful biomarkers for predicting the severity of exacerbation and prognosis of inpatients with AECOPD.
\end{abstract}

Keywords: Chronic obstructive pulmonary disease (COPD); eosinophils (EOS); inflammation; quality of life; respiratory function tests

Submitted Jun 12, 2020. Accepted for publication Nov 12, 2020.

doi: $10.21037 /$ jtd-20-2178

View this article at: http://dx.doi.org/10.21037/jtd-20-2178

\section{Introduction}

Chronic obstructive pulmonary disease (COPD), currently the fourth most common cause of death worldwide, is a heterogeneous disease with complex underlying pathophysiology (1). For a long time, COPD was recognized as a mainly neutrophil-mediated inflammatory disease (2), but recent research has demonstrated that eosinophil-associated airway inflammation is closely related to the occurrence and progress of COPD through damage 
to the physiological structure and function of the airway mucosa, which contains abundant antimicrobial peptides. Consequently, eosinophils (EOS) might be a potential candidate biomarker of inflammation in both stable and acute exacerbations of COPD $(3,4)$. For example, the blood EOS count is already being used as a biomarker of the risk of exacerbation in stable COPD (5), the risk of pneumonia in COPD patients taking inhaled corticosteroids (ICS), and the response to treatment with ICS in patients with COPD (6-8). Data from the WISDOM study of 2,420 patients showed those with EOS $\geq 4 \%$ or using or discontinuing ICS experienced a higher risk of exacerbation in COPD (9). Additionally, the Global Initiative for Chronic Obstructive Lung Disease (GOLD) recommends that blood EOS count could be used as a biomarker to guide ICS therapy in clinical practice $(10,11)$.

Bafadhel et al. reported that patients with blood EOS counts $\geq 200$ cells/mL and/or percentage of blood EOS $\geq 2 \%$ experienced shorter length of stay (LOS) during hospitalization for AECOPD (3). Similarly, longer hospital stays, higher admissions to the intensive care unit (ICU), and increased mortality rates were found in patients with a percentage of blood EOS <2\% (12-14). However, Salturk et al. reported that the percentage of blood EOS $<2 \%$ was associated with shorter ICU stay and lower mortality rates (15). Thus, the association between blood EOS and prognosis, death, change in pulmonary function, and symptoms in AECOPD remains controversial (16).

Therefore, this study aimed to further investigate the association of the EOS level in peripheral blood and inflammatory indicators, arterial blood gas, pulmonary function, need for mechanical ventilation, LOS and mortality rate of patients with COPD. Many studies, such as the survey of the ECLIPSE cohort, have shown a strong correlation between the percentage of blood EOS and absolute blood EOS count $(\mathrm{r}=0.92 ; \mathrm{P}<0.001)(4,17)$. Taking this into account, we classified 174 patients with AECOPD based only on the percentage of blood EOS. The relationship between EOS, inflammation, and clinical data was further studied by grouping patients according to different threshold levels of EOS. We present the following article in accordance with the TREND reporting checklist (available at http://dx.doi.org/10.21037/jtd-20-2178).

\section{Methods}

\section{Materials}

This observational study was conducted in the Department of Respiratory Medicine of the Fourth Affiliated Hospital of Harbin Medical University from January 2018 to November 2019. A total of 211 patients considered for a diagnosis of AECOPD were admitted to the hospital. Among them, 37 patients were excluded from analysis for failing to complete a pulmonary function test (PFT), diagnosis of chronic bronchitis, or for other reasons. All enrolled patients deemed eligible as a subject of the study conformed with the clinical diagnosis of COPD according to the GOLD criteria $(10,11)$. All data were accessed from the hospital's databases or by questionnaires.

The inclusion criteria for patients were the following: (I) aged 41-78 years; (II) no drug or non-drug therapy during the stable stage of disease; (III) routine baseline peripheral blood test results before receiving any antibiotic, or inhaled or systemic corticosteroid therapy; (IV) able to complete PFT.

Meanwhile, the exclusion criteria were the following: (I) severe liver and kidney dysfunction or primary cardiovascular and cerebrovascular disease; (II) bronchial asthma, asthma-COPD overlap syndrome, parasites, or other allergic diseases associated with elevated EOS level in peripheral blood.

For the EOS group, patients were grouped into two major groups for pairwise comparison according to the percentage of blood EOS based on a cutoff value of $2 \%$ : Group 1 (EOS\% <2\%) and Group 2 (EOS\% $\geq 2 \%)$. Group 2 was subdivided into Group A $(2 \% \leq \mathrm{EOS} \%<4 \%)$ and Group B (EOS\% $\geq 4 \%$ ) with $4 \%$ considered the cutoff value.

\section{Study methods}

The study was conducted in accordance with the Declaration of Helsinki (as revised in 2013). After the study was approved by the Institutional Review Board of the Fourth Affiliated Hospital of Harbin Medical University (No. YXLLSC-201904, 2020-SCILLSC-13) and appropriate informed consent from the patients was obtained. Baseline characteristics, including basic information [age, gender, body mass index (BMI), smoking history, heart rate (HR), respiratory rate (RR)], laboratory findings (inflammation indicators and arterial blood gas analysis before receiving any antibiotics, or inhaled or systemic corticosteroid therapy), quality of life assessment test [modified Medical Research Council (mMRC) score, COPD assessment test (CAT) score], PFT, ICU admission rate, duration and utilization rate of noninvasive mechanical 
ventilator (NIMV), LOS and mortality rate during hospitalization, along with rehospitalization rate within 3 years, were summarized and analyzed. This study did not artificially interfere with the patient's individualized treatment such as with antibiotics or bronchodilators (dual $v s$. triple therapy) after collecting the peripheral blood specimen.

The specific measurements were as follows.

(I) Inflammation indicators: data were obtained from the hospital database and included EOS, white blood cell (WBC) count, platelet (PLT) count, neutrophil-to-lymphocyte ratio (NLR), C-reactive protein (CRP), procalcitonin (PCT), and D-dimer.

(II) Arterial blood gas measurement: with the patient assuming a supine position, an arterial blood sample $\left(\mathrm{PaO}_{2}, \mathrm{PaCO}_{2}\right)$ was collected from the radial or femoral artery on admission before any treatment.

(III) PFT: indexes were recorded by professional technicians and measured with MedGraphics Profiler pulmonary function meter within $12 \mathrm{~h}$, and patients were graded into four stages according to the GOLD guideline.

(IV) Assessment of quality of life: mMRC and CAT scores were calculated while patients were hospitalized. In addition, further analysis was conducted in Group 1 (EOS\% <2\%), Group A $(2 \% \leq \mathrm{EOS} \%<4 \%)$, and Group B (EOS\% $\geq 4 \%)$ to compare the different cutoff values of EOS count and inflammation, clinical symptoms and prognosis of COPD. These same three groups were refined and regrouped as mMRC score 1-4 or CAT score 1-4 respectively according to the severity of clinical symptoms or signs (Sx.), including cough and sputum (Sx.1), chest tightness and shortness of breath (Sx.2), dyspnea (Sx.3) and gasp (Sx.4).

\section{Statistical analysis}

Measurement data with a normal distribution, including EOS, CRP, forced vital capacity (FVC), forced expiratory volume in $1 \mathrm{~s}$ (FEV1), FEV1/FVC (\%), FEV1 \% predicted normal (FEV1\%pred), and FVC \% predicted normal (FVC\%pred), were analyzed with $t$-test. Measurement data with a non-normal distribution (WBC and NLR) were analyzed with Mann-Whitney $\mathrm{U}$ test. In addition, invasive and noninvasive mechanical ventilation (IMV, NIMV), inpatient mortality and ICU admission were analyzed with the chi-square test or Fisher's exact test as appropriate.
The correlation of percentage blood EOS to PFT and inflammation indicators was performed via Pearson correlation coefficients. Asterisks $\left({ }^{*}\right)$ denote the statistical significance: * $\mathrm{P}<0.05$, **, $\mathrm{P}<0.01$, and ${ }^{* * *}, \mathrm{P}<0.001$. Data were presented as mean $\pm \mathrm{SD}$. All statistical analyses were conducted using IBM SPSS Statistics 23.0.

\section{Results}

\section{Baseline information}

The 174 screened patients were included in the statistical analysis and classified into four groups according to their blood EOS count: Group 1 (EOS\% <2\%, n=98) and Group 2 (EOS $\% \geq 2 \%, n=76$ ), with Group 2 further divided into Group A ( $\% \leq \mathrm{EOS} \%<4 \%, \mathrm{n}=44)$ and Group B (EOS\% $\geq 4 \%, \mathrm{n}=32$ ) (Figure 1). The average age (mean $\pm \mathrm{SD})$ of the enrolled patients was $65.69 \pm 9.96$ years. There were no significant differences in gender, age, BMI, course of disease, smoking history, HR, or RR (Table 1).

\section{Laboratory data}

Inflammatory indicators and arterial blood gas measurements were significantly different between the two pairs of groups (Group 1 vs. Group 2, Group A vs. Group B), as shown in Table 2. Compared with Group 2, the levels of WBC, NLR, CRP, PCT, and D-dimer were significantly higher in Group $1(\mathrm{P}=0.003,0.003,0.000,0.006$, and 0.032 , respectively). Similarly, significant differences were seen in Groups A and B regarding WBC and CRP, with the latter group's being higher $(\mathrm{P}=0.041, \mathrm{P}=0.000$, respectively). Arterial blood gas analysis showed that patients in Group 2 had higher $\mathrm{PaO}_{2}$ and lower $\mathrm{PaCO}_{2}$ compared with Group $1(71.03 \pm 9.80$ vs. $68.21 \pm 8.86, \mathrm{P}=0.049 ; 40.29 \pm 10.01$ vs. $46.67 \pm 18.22, \mathrm{P}=0.004)$. However, there was no significant difference in PLT between the two pairs of groups (Group 1 vs. Group 2, Group A vs. Group B) ( $>>0.05)$.

\section{Pulmonary function}

The 174 patients were classified into four stages according to the severity of their admission pulmonary function, with 88 of these patients having severe or very severe airflow obstruction (GOLD 3 or GOLD 4). The number and proportion of each pulmonary function classification are shown in Tables 3,4. We found that for GOLD stage 3, the proportion of individuals in Group 1 was $>50 \%$, which 


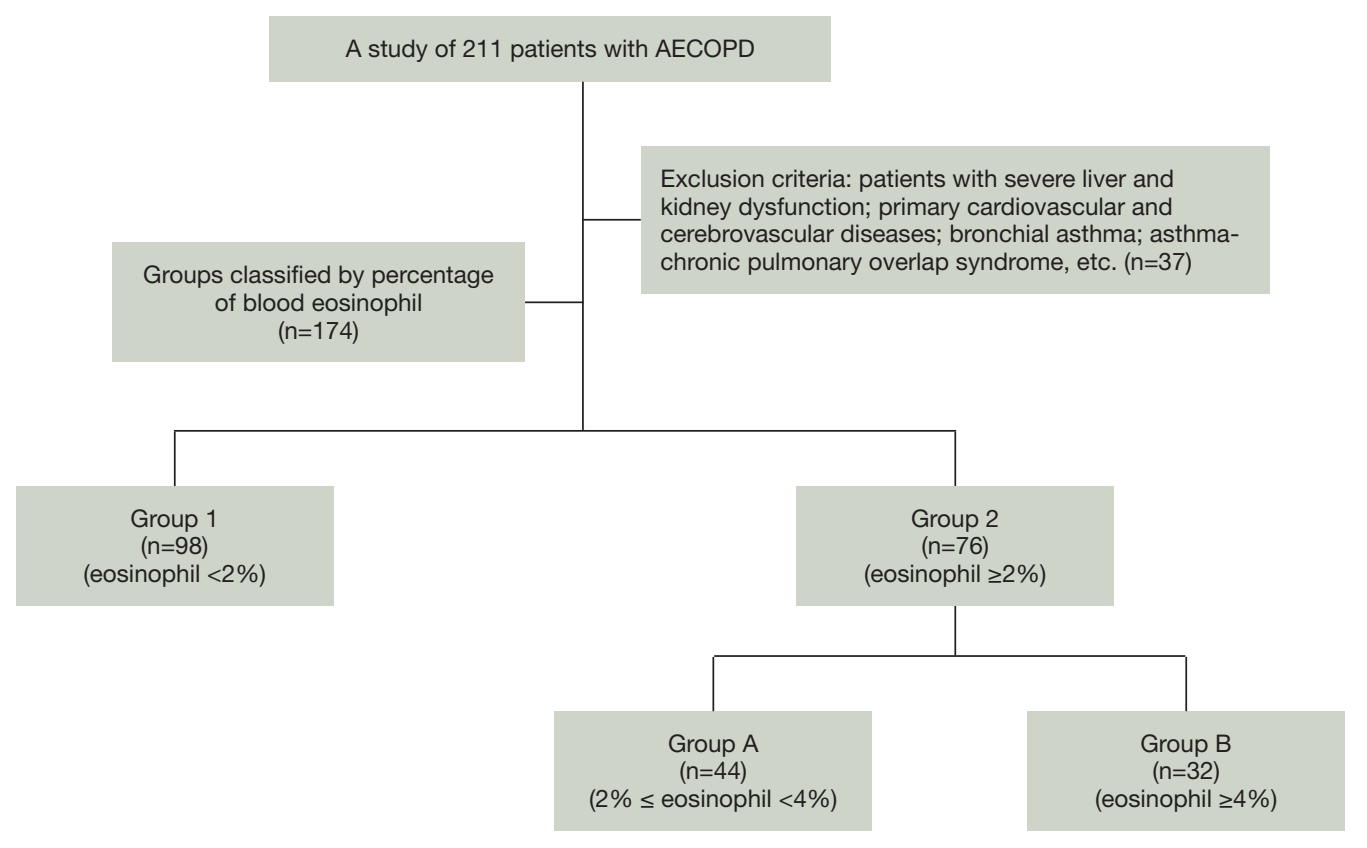

Figure 1 Flow chart of the study.

exceeded that in Group 2. The result was the same for GOLD stage 4. Meanwhile, GOLD 3 (22.03\% vs. 15.26\%) and GOLD 4 (24.14\% vs. 20.69\%) accounted for more patients in Group B than in Group A.

\section{Correlation analysis}

Correlation analyses of EOS to inflammation indicators and PFTs were conducted, and the percentage of total blood EOS was found to be negatively correlated with CRP and NLR $(r=-0.308, \mathrm{P}<0.01 ; \mathrm{r}=-0.227, \mathrm{P}<0.01)$. EOS $\%<2 \%$ was closely related to higher $\mathrm{CRP}(\mathrm{r}=-0.453, \mathrm{P}<0.01)$ (Figure 2), WBC $(\mathrm{r}=-0.225, \mathrm{P}<0.05)$ (Figure $3 A, B)$, and NLR $(\mathrm{r}=-0.407$, $\mathrm{P}<0.01$ ) (Figure $3 C, D$ ) with the correlation coefficient being high, as shown in Table 5. However, the other factors showed no differences. As for pulmonary function, lower FVC\%pred was associated with a high percentage of EOS when $\mathrm{EOS} \% \geq 2 \%$.

\section{Assessment of quality of life}

Clinical survey scales (mMRC and CAT scores) were performed for COPD patients with different percentages of EOS to assess the severity of clinical symptoms, and significant differences were found in the results (Table 3).
Compared with Group 2, Group 1 showed worse mMRC scores $(2.67 \pm 0.99$ vs. $2.33 \pm 0.90, \mathrm{P}=0.019)$ and CAT scores (23.55 \pm 3.54 vs. $22.46 \pm 3.30, \mathrm{P}=0.039)$. Furthermore, compared with Group A, patients in Group B had worse $\mathrm{mMRC}$ and CAT scores $(2.69 \pm 0.82$ vs. $2.07 \pm 0.87, \mathrm{P}=0.003$; $23.34 \pm 3.39$ vs. $21.82 \pm 3.11, \mathrm{P}=0.046)$.

When combined with gradually worsening clinical symptoms of cough and sputum, chest tightness and shortness of breath, dyspnea and gasping, the scores of the two scales in the three ranges of EOS $(<2 \%$ vs. $2-4 \%$ $v s . \geq 4 \%$ ) both increased gradually. The first outcome was that there was no difference in the mMRC and CAT scores among multiple groups (Group 1 vs. Group A; Group 1 vs. Group B; Group A vs. Group B) when only Sx.1 was found. The secondary outcome was that mMRC score 2 in Group A was lower than that in Group B $(1.73 \pm 0.65$ vs. $2.50 \pm 0.53$, $\mathrm{P}=0.013)$ and Group $1(1.73 \pm 0.65$ vs. $2.79 \pm 0.71, \mathrm{P}=0.000)$ when combined with Sx.2. Similarly, a CAT score of 2 in Group A was also lower than that in Group B $(21.64 \pm 3.78$ vs. 22.00 $\pm 3.51, \mathrm{P}=0.834)$ and Group $1(21.64 \pm 3.78$ vs. $23.53 \pm 3.55, \mathrm{P}=0.180$ ) when combined with $\mathrm{Sx} .2$, although this was not significant. In addition, mMRC score 3-4 $(\mathrm{P}=0.036, \mathrm{P}=0.045)$ and CAT score $3-4(\mathrm{P}=0.021, \mathrm{P}=0.041)$ in Group A were also lower than those in Group 1 when Sx.3-4 was experienced (Figure 4, Table 6). 
Table 1 Baseline characteristics of the patients with AECOPD according to blood eosinophil percentage

\begin{tabular}{|c|c|c|c|c|c|}
\hline \multirow{3}{*}{ Variable } & \multirow{3}{*}{ Overall } & \multicolumn{4}{|c|}{ Percentage of blood eosinophils } \\
\hline & & \multirow{2}{*}{ Group 1} & \multicolumn{3}{|c|}{ Group 2} \\
\hline & & & Overall & $A$ & $\mathrm{~B}$ \\
\hline Participants (n) & 174 & 98 & 76 & 44 & 32 \\
\hline \multicolumn{6}{|l|}{ Gender (n) } \\
\hline Male & 97 & 54 & 43 & 25 & 18 \\
\hline Age (years) & $65.69 \pm 9.96$ & $66.43 \pm 10.26$ & $64.74 \pm 9.55$ & $65.61 \pm 10.44$ & $63.53 \pm 8.18$ \\
\hline $\mathrm{BMI}\left(\mathrm{kg} / \mathrm{m}^{2}\right)$ & $22.43 \pm 3.48$ & $22.40 \pm 3.54$ & $22.45 \pm 3.44$ & $22.44 \pm 3.91$ & $22.35 \pm 3.02$ \\
\hline Course of disease, years & $4.33 \pm 2.66$ & $4.23 \pm 2.71$ & $4.45 \pm 2.61$ & $4.41 \pm 2.86$ & $4.50 \pm 2.26$ \\
\hline Ex-smoker & $36(20.69)$ & $18(18.37)$ & 18 (23.69) & $9(20.45)$ & $9(28.13)$ \\
\hline Non-smoker & $88(50.57)$ & $53(54.08)$ & 35 (46.05) & $20(45.45)$ & $15(46.88)$ \\
\hline Smoking index & $319.32 \pm 121.16$ & $324.47 \pm 125.92$ & $313.41 \pm 116.74$ & $314.58 \pm 122.01$ & $311.76 \pm 112.54$ \\
\hline $\mathrm{HR}$ & $78.40 \pm 8.31$ & $78.24 \pm 6.28$ & $78.59 \pm 10.39$ & $77.30 \pm 12.17$ & $80.38 \pm 6.84$ \\
\hline $\mathrm{RR}$ & $20.25 \pm 1.63$ & $20.46 \pm 1.44$ & $19.99 \pm 1.82$ & $19.82 \pm 2.04$ & $20.22 \pm 1.48$ \\
\hline
\end{tabular}

Patients were divided into two groups, with Group 2 further subdivided (Group A and B) by percentage count (2\% and $4 \%$ ) of blood eosinophils. Data were presented as mean $\pm \mathrm{SD}$ or $\mathrm{n}(\%)$. BMI, body mass index; HR, heart rate; $\mathrm{n}$, number; RR, respiratory rate.

Table 2 Comparison of laboratory findings of patients with AECOPD according to percentage count of blood eosinophils

\begin{tabular}{|c|c|c|c|c|c|c|c|}
\hline \multirow{3}{*}{ Variable } & \multirow{3}{*}{ Overall } & \multicolumn{5}{|c|}{ Percentage of blood eosinophils } & \multirow{3}{*}{$P$ value } \\
\hline & & \multirow{2}{*}{ Group 1} & \multicolumn{4}{|c|}{ Group 2} & \\
\hline & & & Overall & A & $\mathrm{B}$ & $P$ value & \\
\hline \multicolumn{8}{|l|}{ Routine blood tests } \\
\hline Eosinophils (\%) & $2.28 \pm 2.30$ & $0.82 \pm 0.62$ & $4.15 \pm 2.32$ & $2.51 \pm 0.46$ & $6.42 \pm 1.90$ & 0.000 & 0.000 \\
\hline NLR & $5.44 \pm 9.22$ & $7.07 \pm 11.83$ & $3.35 \pm 2.66$ & $3.40 \pm 3.12$ & $3.29 \pm 1.90$ & 0.860 & 0.003 \\
\hline Platelets $\left(10^{9} / L\right)$ & $225.02 \pm 72.05$ & $220.00 \pm 69.81$ & $231.51 \pm 74.81$ & $239.23 \pm 80.04$ & $220.91 \pm 66.74$ & 0.295 & 0.297 \\
\hline \multicolumn{8}{|l|}{ Serum laboratory findings } \\
\hline CRP (mg/L) & $15.45 \pm 9.22$ & $18.87 \pm 9.92$ & $11.03 \pm 5.79$ & $8.98 \pm 5.90$ & $13.84 \pm 4.33$ & 0.000 & 0.000 \\
\hline $\mathrm{PaO}_{2}(\mathrm{mmHg})$ & $69.44 \pm 9.36$ & $68.21 \pm 8.86$ & $71.03 \pm 9.80$ & $72.39 \pm 9.62$ & $69.16 \pm 9.89$ & 0.157 & 0.049 \\
\hline $\mathrm{PaCO}_{2}(\mathrm{mmHg})$ & $43.88 \pm 15.48$ & $46.67 \pm 18.22$ & $40.29 \pm 10.01$ & $41.59 \pm 11.78$ & $38.50 \pm 6.67$ & 0.186 & 0.004 \\
\hline $\mathrm{HCO}_{3}^{-}(\mathrm{mmol} / \mathrm{L})$ & $26.22 \pm 5.96$ & $27.12 \pm 7.55$ & $25.11 \pm 2.70$ & $25.05 \pm 2.85$ & $25.19 \pm 2.52$ & 0.823 & 0.017 \\
\hline
\end{tabular}

Patients were divided into two groups, with Group 2 further subdivided (Group A and B) by percentage count ( $2 \%$ and $4 \%$ ) of blood eosinophils. Data were presented as mean \pm SD. CRP, C-reactive protein; NLR, neutrophil-to-lymphocyte ratio; PCT, procalcitonin. 
Table 3 Comparison of clinical outcomes and physiological findings of patients with AECOPD according to percentage count of blood eosinophils

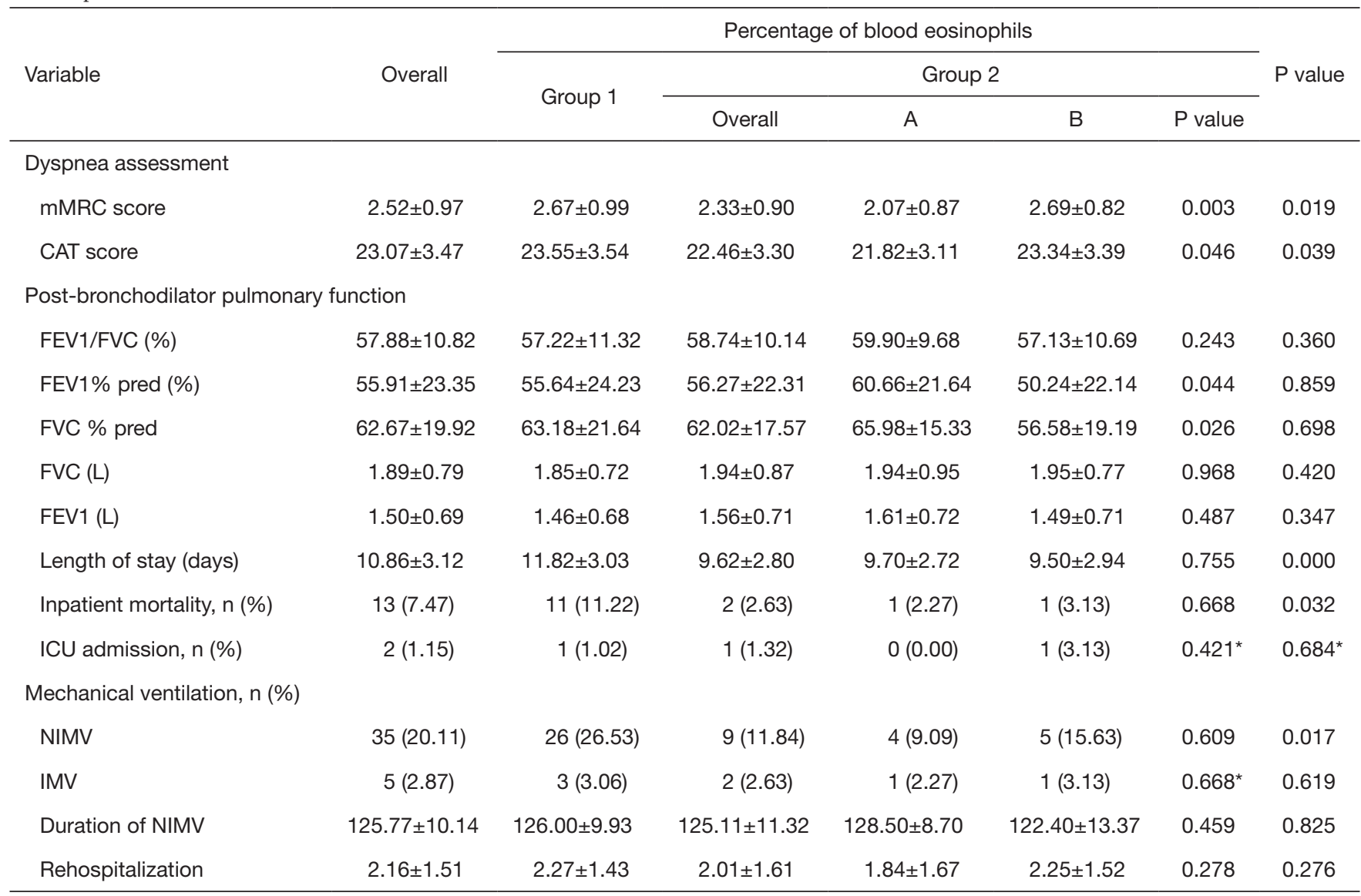

Patients were divided into two groups, with Group 2 further subdivided (Group A and B) by percentage count ( $2 \%$ and $4 \%$ ) of blood eosinophils. *, Fisher's exact probability method. Data were presented as mean \pm SD. CAT, COPD assessment test; FEV1, forced expiratory volume in $1 \mathrm{~s}$; FVC, forced vital capacity; ICU, intensive care unit; IMV, invasive mechanical ventilation; mMRC, modified Medical Research Council; n, number; NIMV, noninvasive mechanical ventilation.

\section{Clinical treatment and prognosis}

Data related to patients' outcomes included the rate of NIMV usage, mortality, ICU admission, rehospitalization and LOS. Higher utilization rate of NIMV $(\mathrm{P}<0.05)$, higher mortality rate $(\mathrm{P}<0.05)$, and longer LOS $(\mathrm{P}<0.001)$ were found in Group 1 compared with Group 2 (Figure 5). However, no significant differences in the rate of ICU and rehospitalization were observed in a pairwise comparison (Group 1 vs. Group B; Group A vs. Group B) (P>0.05).

\section{Discussion}

The aims of this study were to clarify the cutoff values of blood EOS percentage for predicting exacerbation risk and prognosis of AECOPD, and to investigate their correlation with inflammatory indicators and clinical characteristics. The utility of different levels of EOS for assessing the severity and intensity of inflammatory response in patients with COPD has not been evaluated prospectively. Our results from the analysis of low and high EOS percentages may provide practical and potentially meaningful insight into clinically differentiated treatment and medication management.

In this study, EOS was divided according cutoff values of $2 \%$ and $4 \%$, which could more accurately guide the clinical evaluation and prognosis of COPD. Furthermore, we comprehensively analyzed inflammation indicators, pulmonary function, arterial blood gas, respiratory symptoms and the dyspnea index, which produced several noteworthy findings. Our study demonstrated that 
Table 4 GOLD classification of patients with COPD according to percentage count of blood eosinophils

\begin{tabular}{|c|c|c|c|c|c|}
\hline \multirow{2}{*}{ Variable } & \multirow{2}{*}{ Overall } & \multicolumn{4}{|c|}{ Percentage of blood eosinophils } \\
\hline & & Group 1 & Overall & $A$ & $\mathrm{~B}$ \\
\hline Participants (n) & 174 & 98 & 76 & 44 & 32 \\
\hline \multicolumn{6}{|c|}{ GOLD classification, n (\%) } \\
\hline GOLD 2 & 48 & $21(43.75 \%)$ & $27(56.25 \%)$ & $21(43.75 \%)$ & $6(12.50 \%)$ \\
\hline GOLD 3 & 59 & $37(62.71 \%)$ & $22(37.29 \%)$ & $9(15.26 \%)$ & $13(22.03 \%)$ \\
\hline GOLD 4 & 29 & $16(55.17 \%)$ & $13(44.83 \%)$ & $6(20.69 \%)$ & 7 (24.14\%) \\
\hline
\end{tabular}

Patients were divided into two groups with Group 2 further subdivided (Group A and B) by percentage count ( $2 \%$ and $4 \%$ ) of blood eosinophils. GOLD, Global Initiative for Chronic Obstructive Lung Disease; n, number.
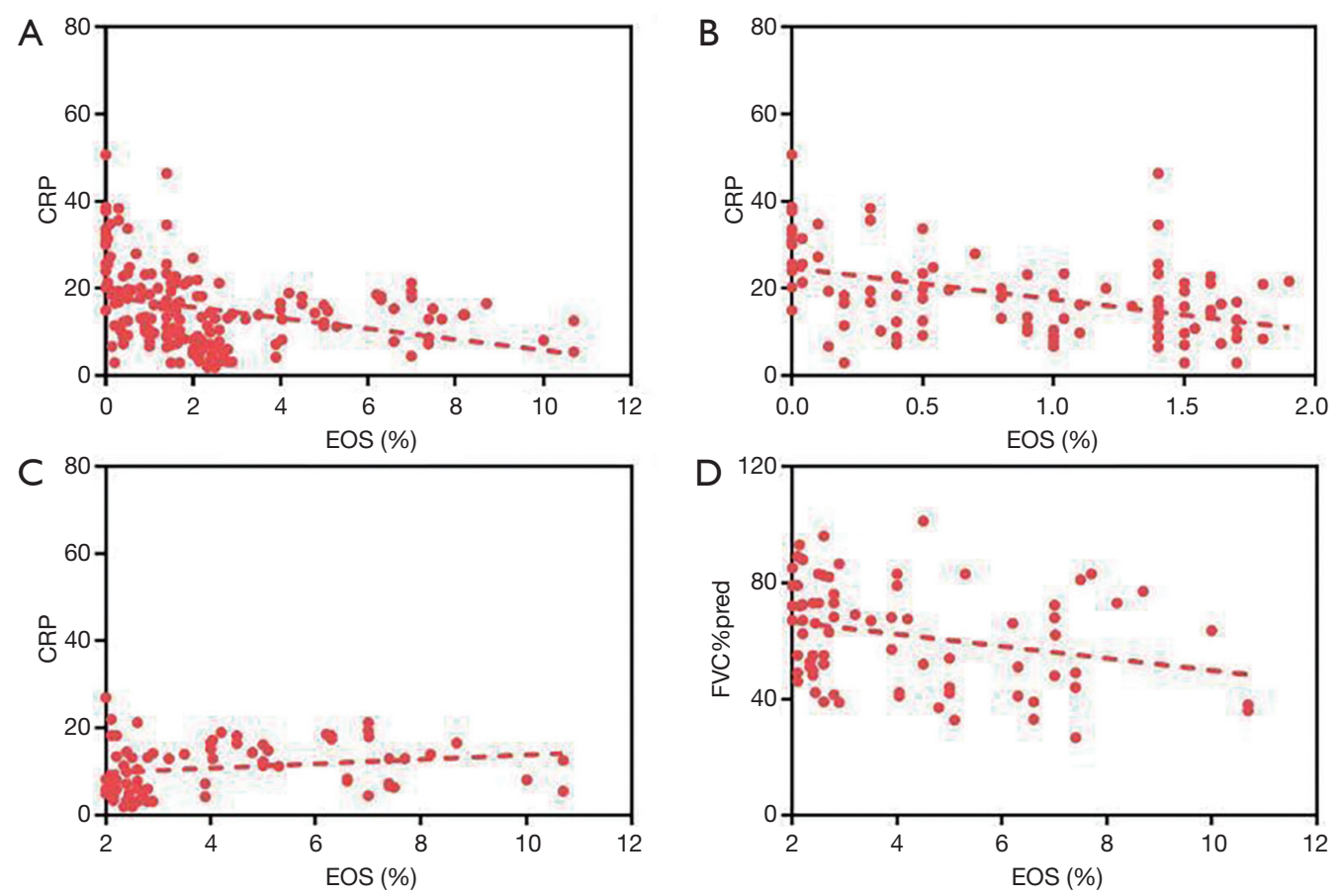

Figure 2 Relationship between eosinophils (EOS), C-reactive protein (CRP), and forced vital capacity \% predicted normal (FVC\%pred). (A) Correlation between total EOS count and CRP ( $\mathrm{r}=-0.308$ and $\mathrm{P}<0.01)$. (B) Correlation between EOS count $(\mathrm{EOS}<2 \%)$ and $\mathrm{CRP}(\mathrm{r}=-0.453$ and $\mathrm{P}<0.01)$. (C) Correlation between EOS count (EOS $\geq 2 \%$ ) and $\mathrm{CRP}(\mathrm{r}=0.258$ and $\mathrm{P}<0.05)$. (D) Correlation between EOS count (EOS $\geq 2 \%)$ and FVC\%pred $(\mathrm{r}=-0.274$ and $\mathrm{P}<0.05)$.

patients could exhibit severe clinical symptoms and high inflammation index not only with EOS $<2 \%$, but also with EOS $\geq 4 \%$, which differs from previous research. Our results suggest that low or high expression of EOS is a manifestation of a dysfunctional immune response, and a reflection of the direct or indirect participation of EOS in the pathogenesis and progression of COPD.

Studies have shown that there is eosinophil-associated airway inflammation in $20-40 \%$ of COPD cases. However, the relationship between blood EOS levels and the prognosis of patients with AECOPD remains controversial. Multiple studies have shown that a decrease in the blood 

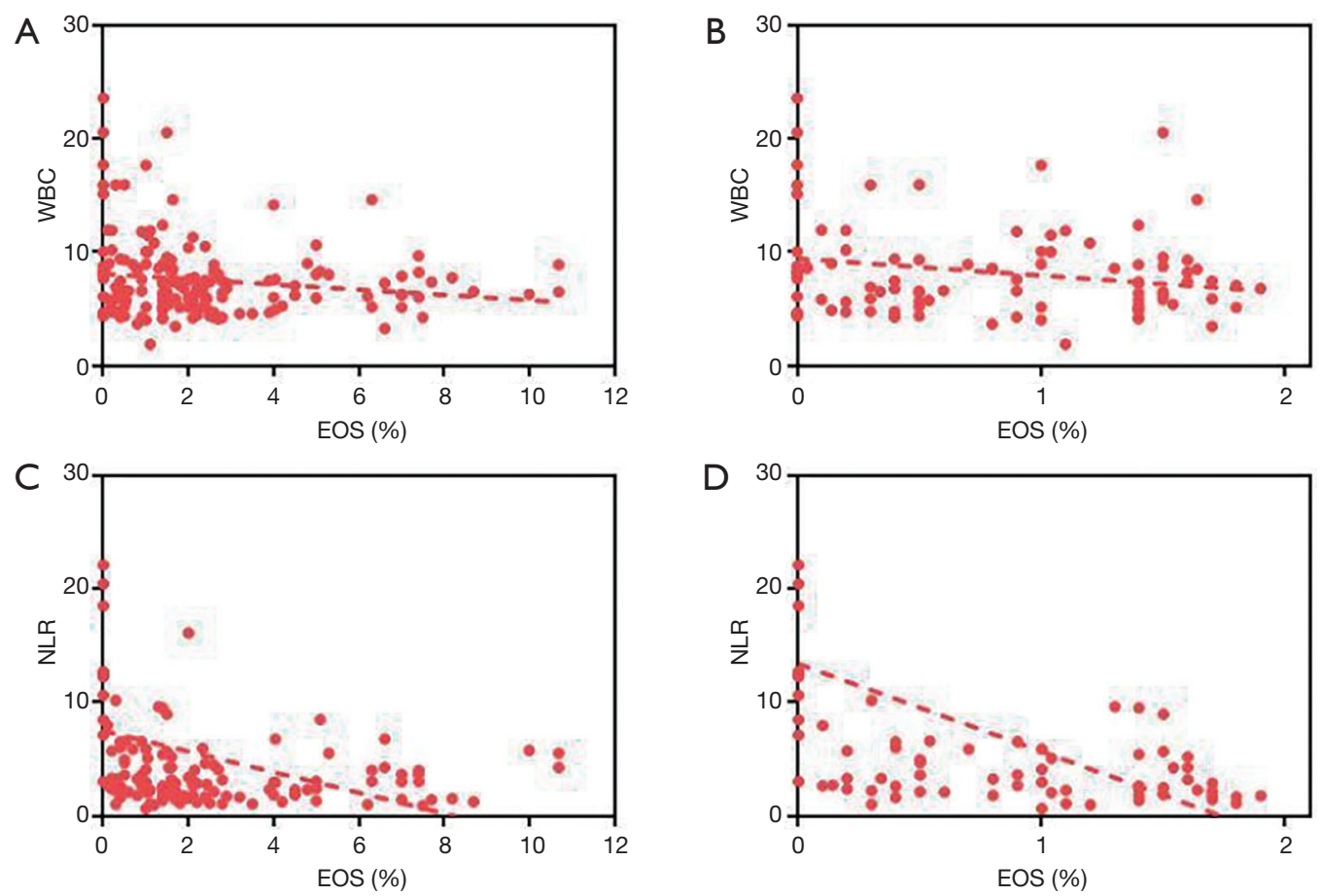

Figure 3 Relationship between EOS, WBC, and NLR. (A) Correlation between total EOS count and WBC ( $\mathrm{r}=-0.155$ and $\mathrm{P}<0.05$. (B) Correlation between EOS count (EOS $<2 \%)$ and WBC $(r=-0.225$ and $\mathrm{P}<0.05$. (C) Correlation between total EOS count and NLR ( $r=-0.227$ and $\mathrm{P}<0.01$. (D) Correlation between EOS count (EOS $<2 \%$ ) and NLR ( $\mathrm{r}=-0.407$ and $\mathrm{P}<0.01)$. EOS, eosinophils; WBC, white blood cells; NLR, neutrophil-to-lymphocyte ratio.

Table 5 Factors associated with percentage count of blood eosinophils according to Pearson correlation coefficients

\begin{tabular}{|c|c|c|c|c|c|c|c|c|}
\hline EOS (\%) & CRP & WBC & NLR & FVC & FVC \% pred & FEV1 & FEV1 \% pred & $\mathrm{FEV}_{1} / \mathrm{FVC}$ \\
\hline EOS $<2 \%$ & $-0.453^{\star \star}$ & $-0.225^{\star}$ & $-0.407^{\star *}$ & 0.162 & 0.122 & 0.016 & 0.054 & 0.102 \\
\hline $2 \% \leq \mathrm{EOS}<4 \%$ & -0.094 & -0.180 & -0.284 & -0.116 & -0.117 & 0.163 & 0.140 & -0.121 \\
\hline$E O S \geq 4 \%$ & -0.318 & -0.111 & 0.121 & 0.119 & -0.108 & 0.108 & 0.012 & -0.040 \\
\hline
\end{tabular}

*, $\mathrm{P}<0.05 ;{ }^{* \star}, \mathrm{P}<0.01$.

EOS level may be associated with a poor prognosis. For example, Bafadhel et al. reported that, among patients with AECOPD, those with EOS $<2 \%$ experienced longer LOS, more mechanical ventilation needs and a higher mortality rate $(3,18)$. Also, the SPIROMICS study suggested worse pulmonary function and severe airflow restriction in patients with lower blood EOS level $(8,19)$. Thus, we can't evaluate the disease condition of patients with diverse clinical manifestations only based on pulmonary function alone.
On the other hand, some researchers showed that patients with EOS $\geq 2 \%$ had a higher risk of AECOPD $(15,20)$, while another study did not find a relationship between blood EOS level and lung function, clinical symptoms, and risk of exacerbation in a COPD cohort. Data from the WISDOM study of 2,420 cases showed patients with EOS $\geq 4 \%$ using or discontinuing ICS experienced a higher risk of exacerbation in COPD. However, other studies reported that the use of ICS could decrease the blood EOS count in patients with AECOPD $(21,22)$ 


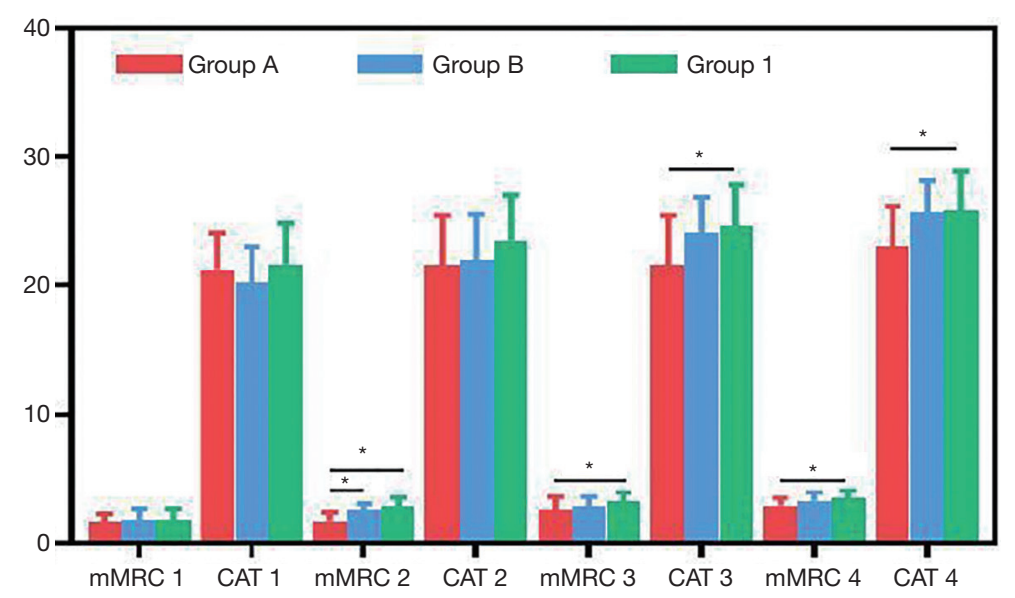

Figure 4 Comparison of CAT and mMRC scores of different clinical symptoms in Group A, Group B, and Group 1. *, statistical significance: $\mathrm{P}<0.05$. Data were presented as mean $\pm \mathrm{SD}$. CAT, COPD assessment test; mMRC, modified Medical Research Council.

Table 6 Comparison of CAT and mMRC scores of different clinical symptoms in Groups A, B, and 1

\begin{tabular}{|c|c|c|c|}
\hline Clinical symptoms & \multicolumn{3}{|c|}{ Groups } \\
\hline mMRC score 1 & $1.83 \pm 0.82$ & $1.63 \pm 0.62$ & $1.80 \pm 0.84$ \\
\hline CAT score 1 & $21.71 \pm 3.11$ & $21.19 \pm 2.88$ & $20.20 \pm 2.86$ \\
\hline mMRC score 2 & $2.79 \pm 0.71$ & $1.73 \pm 0.65$ & $2.50 \pm 0.53$ \\
\hline CAT score 2 & $23.53 \pm 3.55$ & $21.64 \pm 3.78$ & $22.00 \pm 3.51$ \\
\hline \multicolumn{4}{|l|}{ Expiratory dyspnea } \\
\hline mMRC score 3 & $3.21 \pm 0.74$ & $2.50 \pm 1.07$ & $2.82 \pm 0.75$ \\
\hline mMRC score 4 & $3.44 \pm 0.63$ & $2.89 \pm 0.60$ & $3.25 \pm 0.71$ \\
\hline CAT score 4 & $25.81 \pm 3.08$ & $23.00 \pm 3.20$ & $25.63 \pm 2.56$ \\
\hline
\end{tabular}

Patients were divided into two groups, with Group 2 further subdivided (Group A and B) by percentage count (2\% and $4 \%$ ) of blood eosinophils. Data were presented as mean \pm SD. CAT, COPD assessment test; mMRC, modified Medical Research Council.

through enhancing adhesion between EOS and the vascular endothelium. These conflicting results may be related to the use of ICS before sample collection or a variation in clinical features of AECOPD patients including population genetics, living environment etc. Taken together, these findings do not indicate a clear significance for the cutoff value of EOS in the progression and prognosis of COPD. Moreover, matters such as the severity of inflammatory indicators, the quality of airway management and the risk of death in COPD patients with EOS $\geq 4 \%$, and whether a higher EOS correlates with milder symptoms, have remained unresolved. Therefore, $2 \%$ and $4 \%$ were accepted as the cutoff values of EOS in this study to analyze their significance in COPD.

Our data demonstrated that different EOS grouping definition standards were accompanied by different 

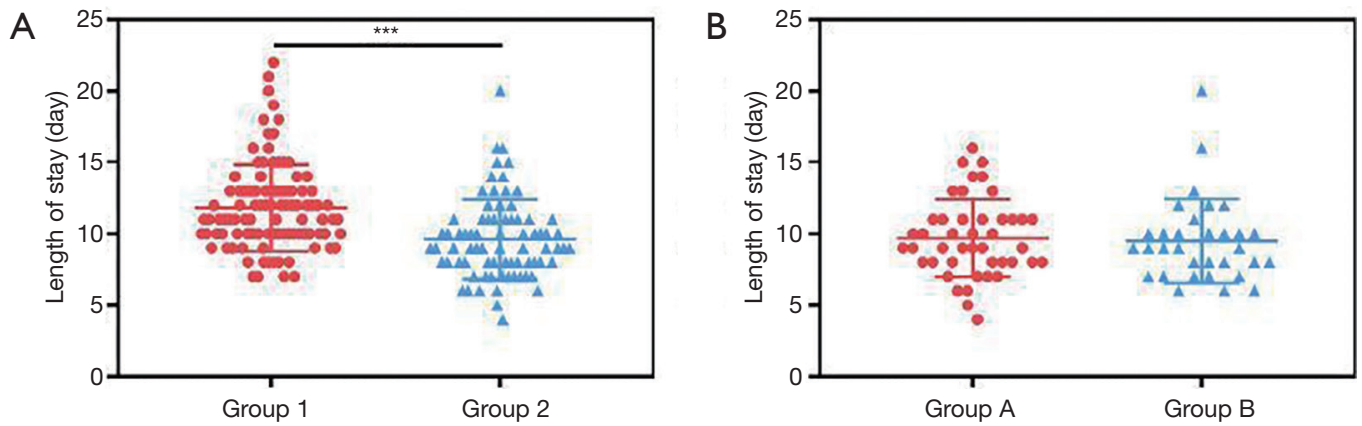

Figure 5 Comparison of the length of stay (LOS) in hospital between different groups. (A) Statistical analysis of the LOS in relation to the different eosinophil (EOS) cut-off values. Group 1: EOS $<2 \%$; Group 2: EOS $\geq 2 \%$. (B) Statistical analysis of the LOS in relation to the different EOS cutoff values. Group A: $2 \% \leq \mathrm{EOS}<4 \%$; Group B: EOS $\geq 4 \%$. ${ }^{* * *}, \mathrm{P}<0.001$. Data were presented as mean \pm SD.

conclusions, suggesting that biological markers need to be properly understood and analyzed in clinical care. The experimental results showed that the percentage of blood EOS has further significance for guiding the prognosis of patients with AECOPD. When the patients were initially divided into Group 1 (EOS $<2 \%$ ) and Group 2 (EOS $\geq 2 \%$ ), those with $\mathrm{EOS}<2 \%$ showed higher inflammatory indices (WBC, CRP, NLR, PCT), more severe clinical dyspnea, more pronounced hypoxia, relatively high carbon dioxide retention, longer hospital stay, and higher mortality rate compared with AECOPD patients with EOS $\geq 2 \%$. Increased CRP and NLR often indicate a severe inflammatory response, and PCT often points to a high possibility of bacterial infection. Meanwhile, an increase of D-dimer may indirectly reflect an increase in vascular blood viscosity, suggesting a risk of vascular embolism, although there was no significant difference in PLT between Groups 1 and 2. EOS, as a main component of the immune system, may assist in eliminating pathogens and harmful metabolites, thereby promoting tissue repair when the immune system is activated. The defense ability of the host could be affected and the permeability of blood vessels might change when the EOS level decreases. Therefore, it could be speculated that AECOPD patients with EOS $<2 \%$ generally suffer more severe airway inflammation during acute exacerbations and have a worse prognosis, which is also accompanied by an increased risk of complications such as vascular inflammatory diseases and infection.

However, when Group 2 (EOS $\geq 2 \%$ ) was further subdivided into Group A $(2 \% \leq \mathrm{EOS}<4 \%)$ and Group B (EOS $\geq 4 \%$ ) with a cutoff value of $4 \%$, the inflammation index CRP was higher in Group B than in Group A
$(\mathrm{P}<0.05)$, which was contrary to the pattern described above. Similarly, higher $\mathrm{mMRC}$ and CAT scores were observed in the group with EOS $>4 \%$, which was associated with worse clinical symptoms. Moreover, this study also found that the proportion of patients with poor pulmonary function in Group B was higher than that in group A for GOLD stage 3-4. Meanwhile, EOS negatively correlated with $\mathrm{FVC} \%$ pred when $\mathrm{EOS} \geq 2 \%$, suggesting that high EOS indicated poor pulmonary function. These are associated with an immune response mediated by cytotoxic substances and metabolites which occurs when EOS continue to increase. Overexpression of EOS might lead to the damage of the airway mucosal structure and function, invasion and reproduction of pathogens, aggravation of airway remodeling, contraction and increased reactivity of smooth muscle, and accumulation of sputum, thus aggravating the clinical symptom of dyspnea in patients. However, the $\mathrm{mMRC}$ and CAT scores in Group 1 (EOS $<2 \%$ ) and Group B (EOS $\geq 4 \%$ ) were both higher, which suggested that an increase or decrease of EOS might be associated with severe clinical symptoms. Therefore, it cannot be simply assumed that when $\operatorname{EOS} \geq 2 \%$, the patient's clinical condition is mild. Over-expression or under-expression of EOS is a manifestation of a dysfunctional immune response, indicating a disruption in the balance of the microenvironment.

A few limitations to this study should also be addressed. Firstly, it was a single-center and small-sample study, with no significant differences in patient demographics. Secondly, we did not collect and evaluate EOS in the patient's sputum, so it was difficult to directly analyze the relationship between EOS in the peripheral blood and the EOS in the airway. Thirdly, our study cannot directly 
explain the involvement of EOS in the mechanism of the pathogenesis and progression of COPD. Fourthly, the subjects in our study had no or few comorbidities when they were admitted to hospital, so there exist certain limitations concerning the representativeness of the research subjects, and further studies need to be performed. We will continue to collect and record the clinical features, inflammation indicators, LOS, mortality rate, and other relevant data of patients with COPD, and will conduct a multicenter study to further explore the relationship between EOS and COPD, as well as their potential significance to clinical treatment. Meanwhile, we will link sputum EOS with peripheral blood EOS in a more detailed analysis that may better clarify changes in airway inflammation.

In summary, the percentage of blood EOS was associated with AECOPD status. Recent evidence has shown that COPD patients with low EOS have a higher risk of acute attack, but few studies of high EOS have been conducted. More detailed clinical studies of different cutoff values of EOS in COPD need to be carried out. If this biomarker could be understood and correctly used to evaluate airway inflammation changes, severity of disease, risk of deterioration and prognosis of patients, it will greatly assist with rational drug selection, and individualized therapy. The rate of recurrence, disability, and mortality of patients with AECOPD might thus be greatly reduced.

\section{Conclusions}

Patients with a lower level of EOS showed severe inflammation, longer LOS, increased mortality rate, and higher CAT and mMRC scores, which suggested worse prognosis. However, when $\mathrm{EOS} \geq 4 \%$, the clinical symptoms and inflammatory indices of the patients were also severe. Different cutoff values of blood EOS might be useful biomarkers for predicting outcomes and prognosis of patients with AECOPD.

\section{Acknowledgments}

Funding: This work was supported by the National Natural Science Foundation of China [81670028] and the Graduate Practice Innovation Project Fund of Harbin Medical University [2018182].

\section{Footnote}

Reporting Checklist: The authors have completed the
TREND reporting checklist. Available at http://dx.doi. org/10.21037/jtd-20-2178

Data Sharing Statement: Available at http://dx.doi. org/10.21037/jtd-20-2178

Conflicts of Interest: All authors have completed the ICMJE uniform disclosure form (available at http://dx.doi. org/10.21037/jtd-20-2178). The authors have no conflicts of interest to declare.

Ethical Statement: The authors are accountable for all aspects of the work in ensuring that questions related to the accuracy or integrity of any part of the work are appropriately investigated and resolved. The study was conducted in accordance with the Declaration of Helsinki (as revised in 2013). The study was approved by the Institutional Review Board of the Fourth Affiliated Hospital of Harbin Medical University (No. YXLLSC-201904, 2020-SCILLSC-13) and informed consent from the patients was obtained.

Open Access Statement: This is an Open Access article distributed in accordance with the Creative Commons Attribution-NonCommercial-NoDerivs 4.0 International License (CC BY-NC-ND 4.0), which permits the noncommercial replication and distribution of the article with the strict proviso that no changes or edits are made and the original work is properly cited (including links to both the formal publication through the relevant DOI and the license). See: https://creativecommons.org/licenses/by-nc-nd/4.0/.

\section{References}

1. Barnes PJ, Burney PG, Silverman EK, et al. Chronic obstructive pulmonary disease. Nat Rev Dis Primers 2015;1:15076.

2. Brusselle G, Pavord I D, Landis S, et al. Blood eosinophil levels as a biomarker in COPD. Respir Med 2018;138:21-31.

3. Bafadhel M, McKenna S, Terry S, et al. Acute Exacerbations of Chronic Obstructive Pulmonary Disease. Am J Respir Crit Care Med 2011;184:662-71.

4. Singh D, Kolsum U, Brightling CE, et al. Eosinophilic inflammation in COPD: prevalence and clinical characteristics. Eur Respir J 2014;44:1697.

5. Oh YM, Lee KS, Hong Y, et al. Blood eosinophil count as a prognostic biomarker in COPD. Int J Chron Obstruct 
Pulmon Dis 2018;13:3589-96.

6. Pascoe S, Locantore N, Dransfield MT, et al. Blood eosinophil counts, exacerbations, and response to the addition of inhaled fluticasone furoate to vilanterol in patients with chronic obstructive pulmonary disease: a secondary analysis of data from two parallel randomised controlled trials. Lancet Respir Med 2015;3:435-42.

7. Barnes N C, Sharma R, Lettis S, et al. Blood eosinophils as a marker of response to inhaled corticosteroids in COPD. Eur Respir J 2016;47:1374-82.

8. Hastie AT, Martinez FJ, Curtis JL, et al. Association of sputum and blood eosinophil concentrations with clinical measures of COPD severity: an analysis of the SPIROMICS cohort. Lancet Respir Med 2017;5:956-67.

9. Watz H, Tetzlaff K, Wouters EFM, et al. Blood eosinophil count and exacerbations in severe chronic obstructive pulmonary disease after withdrawal of inhaled corticosteroids: a post-hoc analysis of the WISDOM trial. Lancet Respir Med 2016;4:390-8.

10. Mirza S, Clay RD, Koslow MA, et al. COPD Guidelines: A Review of the 2018 GOLD Report. Mayo Clin Proc 2018;93:1488-502.

11. Muneswarao J, Verma AK, Hassali MAA. Global initiative for chronic obstructive lung disease (GOLD) 2018 report: Highlighting an incorrect information. Pulm Pharmacol Ther 2018;49:10.

12. MacDonald MI, Osadnik CR, Bulfin L, et al. Low and High Blood Eosinophil Counts as Biomarkers in Hospitalized Acute Exacerbations of COPD. Chest 2019;156:92-100.

13. Holland M, Alkhalil M, Chandromouli S, et al. Eosinopenia as a marker of mortality and length of stay in patients admitted with exacerbations of chronic obstructive pulmonary disease. Respirology 2010;15:165-7.

14. Ko FWS, Chan KP, Ngai J, et al. Blood eosinophil

Cite this article as: Lv MY, Qiang LX, Li ZH, Jin SD. The lower the eosinophils, the stronger the inflammatory response? The relationship of different levels of eosinophils with the degree of inflammation in acute exacerbation chronic obstructive pulmonary disease (AECOPD). J Thorac Dis 2021;13(1):232-243. doi: 10.21037/jtd-20-2178 count as a predictor of hospital length of stay in COPD exacerbations. Respirology 2020;25:259-66.

15. Salturk C, Karakurt Z, Adiguzel N, et al. Does eosinophilic COPD exacerbation have a better patient outcome than non-eosinophilic in the intensive care unit? Int J Chron Obstruct Pulmon Dis 2015;10:1837-46.

16. Wu HX, Zhuo KQ, Cheng DY. Peripheral Blood Eosinophil as a Biomarker in Outcomes of Acute Exacerbation of Chronic Obstructive Pulmonary Disease. Int J Chron Obstruct Pulmon Dis 2019;14:3003-15.

17. Vestbo J, Anderson W, Coxson HO, et al. Evaluation of COPD Longitudinally to Identify Predictive Surrogate End-points (ECLIPSE). Eur Respir J 2008;31:869-73.

18. Rahimi-Rad MH, Asgari B, Hosseinzade N, et al. Eosinopenia as a Marker of Outcome in Acute Exacerbations of Chronic Obstructive Pulmonary Disease. Maedica 2015;10:10-3.

19. Weir M, Zhao H, Han MK, et al. Eosinophils In Chronic Obstructive Pulmonary Disease, The SPIROMICS Cohort. Available online: https://www.atsjournals. org/doi/abs/10.1164/ajrccm-conference.2014.189.1_ MeetingAbstracts.A5902

20. Vedel-Krogh S, Nielsen SF, Lange P, et al. Blood Eosinophils and Exacerbations in Chronic Obstructive Pulmonary Disease. The Copenhagen General Population Study. Am J Respir Crit Care Med 2016;193:965-74.

21. Tashkin DP, Strange C. Inhaled corticosteroids for chronic obstructive pulmonary disease: what is their role in therapy? Int J Chron Obstruct Pulmon Dis 2018;13:2587-601.

22. Vanfleteren L, Fabbri LM, Papi A, et al. Triple therapy (ICS/LABA/LAMA) in COPD: time for a reappraisal. Int J Chron Obstruct Pulmon Dis 2018;13:3971-81. 